\title{
High-dose-rate intraoperative radiation therapy: the nuts and bolts of starting a program
}

\author{
Shalini Moningi, BA', Elwood P. Armour, PhD, DABR', Stephanie A. Terezakis, MD!', Jonathan E. Efron, MD², \\ Susan L. Gearhart, MD², Trinity J. Bivalacqua, MD, PhD³, Rachit Kumar, MD!, Yi Le, PhD', Sook Kien Ng, PhD! \\ Christopher L. Wolfgang, MD, PhD², Richard C. Zellars, MD!, Susannah G. Ellsworth, MD', Nita Ahuja, MD², \\ Joseph M. Herman, MD, MSc' \\ 'Department of Radiation Oncology \& Molecular Radiation Sciences, ${ }^{2}$ Department of Surgery, ${ }^{3}$ Department of Urology, The Sidney Kimmel \\ Comprehensive Cancer Center, Johns Hopkins Hospital, Baltimore, MD, USA
}

\begin{abstract}
High-dose-rate intraoperative radiation therapy (HDR-IORT) has historically provided effective local control (LC) for patients with unresectable and recurrent tumors. However, IORT is limited to only a few specialized institutions and it can be difficult to initiate an HDR-IORT program. Herein, we provide a brief overview on how to initiate and implement an HDR-IORT program for a selected group of patients with gastrointestinal and pelvic solid tumors using a multidisciplinary approach. Proper administration of HDR-IORT requires institutional support and a joint effort among physics staff, oncologists, surgeons, anesthesiologists, and nurses. In order to determine the true efficacy of IORT for various malignancies, collaboration among institutions with established IORT programs is needed.
\end{abstract}

Key words: high-dose-rate brachytherapy, intraoperative radiation therapy, recurrent tumors, sarcoma.

\section{Purpose}

Over the past two decades, intraoperative radiation therapy (IORT) has become an increasingly utilized modality for the treatment of breast, head \& neck, pelvic, and gastrointestinal malignancies [1-6]. Several studies have demonstrated improved local control, especially in margin-negative patients [1,7-9]. While external beam radiation therapy (EBRT) is the standard modality utilized to treat locally advanced or recurrent solid tumors, there are additional benefits associated with IORT. For example, a single dose of IORT has approximately 2.5 fold more biological effectiveness than an equivalent dose of radiation delivered via EBRT (Table 1) [7,10].

While IORT can be used alone, it is unlikely that it will deliver a biological equivalent dose that is sufficient to kill microscopic disease [7]. Therefore, IORT following neoadjuvant radiation therapy (RT), in which the tumor itself displaces organs-at-risk (OARs), provides optimal results. Adjuvant EBRT can be delivered following IORT; however, normal tissues such as the bowel may enter the radiation field, thus increasing toxicity [4,7]. IORT can be delivered by using Linac-based electron beam radiation therapy (IOERT), low-energy photons, or by highdose-rate brachytherapy catheters (HDR-IORT). All of these techniques deliver high doses of radiation to the target site while protecting normal surrounding struc- tures through retraction or shielding using lead sheets. With regard to dose distribution, HDR-IORT can deliver a more concentrated dose at the surface of the target area while IOERT delivers radiation dose more homogenously throughout the tissue depth $[8,11]$. A major advantage of HDR-IORT over IOERT and IORT using low-energy photons lies in its delivery method. HDR-IORT uses flexible and personalized applicators (Harrison-Anderson-Mick [HAM] applicator or Freiburg Flap) to deliver RT in contrast to the more rigid cone applicators used with both IOERT and IORT with low-energy photons [8,9]. The flexible Freiburg applicator used in HDR-IORT is a silicone flap that can be re-shaped to accurately resemble the shape of the tumor bed [12]. The flap is particularly helpful in situations where the tumor is located in difficult locations such as abutting vasculature or deep in the pelvis where it might be difficult to place the cone needed to deliver IOERT. This article will focus primarily on the initiation and development of an HDR-IORT program, its multidisciplinary approach, patient selection, and its use in patients with gastrointestinal and pelvic solid tumors.

The vast majority of studies utilizing IORT have been retrospective, single institution reviews. IORT has been associated with improved local control (LC) in several malignancies, especially when combined with neoadjuvant or adjuvant EBRT. For example, IORT in conjunction with surgical resection and EBRT has shown favorable
Address for correspondence: Joseph M. Herman, MD, MSc, Department of Radiation Oncology \& Molecular Radiation Sciences, Sidney Kimmel Comprehensive Cancer Center, Johns Hopkins Hospital, 401 N. Broadway, Weinberg Suite 1440, Baltimore, MD 21231, USA, phone: +1 410-955-6980, fax: +1 410-502-1419, « e-mail: jherma15@jhmi.edu
Received: 31.12 .2013

Accepted: 01.03.2014

Published: 28.03.2014 
Table 1. Estimated equivalent EBRT doses of varying IORT doses [10]

\begin{tabular}{lccc} 
IORT dose & $\begin{array}{c}\text { EBRT normal } \\
\text { tissue dose } \\
\text { (acute) }\end{array}$ & $\begin{array}{c}\text { EBRT tumor } \\
\text { dose }\end{array}$ & $\begin{array}{c}\text { EBRT normal } \\
\text { tissue dose } \\
\text { (late) }\end{array}$ \\
\hline $10 \mathrm{~Gy}$ & $20 \mathrm{~Gy}$ & $17 \mathrm{~Gy}$ & $30 \mathrm{~Gy}$ \\
\hline $15 \mathrm{~Gy}$ & $37 \mathrm{~Gy}$ & $31 \mathrm{~Gy}$ & $65 \mathrm{~Gy}$
\end{tabular}

5-year LC when compared to EBRT alone in the treatment of adult sarcomas [4,7]. Similarly, favorable LC rates were seen with the use of IORT along with maximal surgical resection for recurrent cervical cancer, recurrent colorectal cancer, pancreatic cancer, and other solid malignancies [2-9].

Patients with locally advanced or recurrent solid tumors are at a high risk of local progression and poor outcomes. The addition of IORT to standard treatment paradigms for locally advanced tumors may result in improved LC and survival while limiting treatment-related toxicity $[1-8,10]$. However, IORT is not readily available at most cancer centers due to the need for specialized equipment and a shielded operating room [8]. Furthermore, limited information is available on how to implement an IORT program and how to ascertain which patients are most likely to benefit from IORT. After the successful implementation of our IORT program at Johns Hopkins Hospital, we provide the first report on how to initiate and implement an HDR-IORT program and how to select optimal patients for treatment with HDR-IORT. Finally, we provide two patient examples to elucidate how to approach standard and complex cases.

\section{Material and methods}

\section{Initiation of an intraoperative radiation therapy program}

Implementation of a new IORT program requires access to technology and equipment (ie. shielded operating rooms, HDR afterloaders, treatment planning software, etc.), institutional funding, interdepartmental planning, and communication, development of an Institution Review Board (IRB) approved database or registry of patients, development of a multidisciplinary training program, and simulations of IORT treatment delivery in the operating room (OR) to ensure that procedures are followed safely.

In 2006, the Johns Hopkins Department of Radiation Oncology, in collaboration with the Weinberg Perioperative Clinical Services Team, introduced HDR-IORT to the institution [13]. A systematic approach was developed to safely deliver IORT through multiple methods including workflow grids (Fig. 1).

Due to the complex and multidisciplinary nature of HDR-IORT, numerous safety simulations and risk assessment workshops were convened. A checklist to effectively deliver therapy while minimizing risks was developed and is now part of the HDR-IORT protocol (Fig. 2) [13]. Proper training and close collaboration between radiation oncology, surgery, surgical pathology, anesthesio- logy, medical physics, and the nursing staff is essential for safe and effective management of the patient during HDR-IORT $[13,14]$.

\section{Patient selection}

Appropriate patient selection for HDR-IORT should occur in a multidisciplinary setting. Several criteria should be taken into consideration when assessing the role of HDR-IORT for a patient [11,12]. The following considerations are particularly important when selecting patients who can benefit from HDR-IORT:

1) based on preoperative imaging, if the attempted surgical resection would likely result in positive or close margins,

2) when EBRT does not achieve satisfactory LC,

3) during treatment planning, the dose needed to reach optimal control cannot be obtained due to dose tolerance of surrounding OARs, such as bowel and bladder,

4) if HDR-IORT can be used instead of EBRT following a conservative surgical resection,

5) if HDR-IORT can be used in addition to neoadjuvant EBRT to achieve high LC rates and minimize toxicity to organs at risk (OARs).

\section{Intraoperative radiation therapy delivery technique}

HDR-IORT delivery requires high-dose-rate after-loader technology combined with a commercially available flexible flap applicator (Freiburg Flap ${ }^{\circledR}$, Nucletron, an Elekta company, Elekta AB, Stockholm, Sweden) to deliver uniform dose to the tissue surface or to a specified depth below the surface. The Freiburg Flap consists of linear, connected silicone rubber balls forming a planar flexible sheet through, which 6-French after-loading catheters can be inserted. The size and shape of the area can be customizable to any grid pattern with a one $\mathrm{cm}$ resolution.

The high-risk area of the surgical bed is identified and measured in collaboration with the surgical oncologist, and the Freiburg flap is cut to size once the target area is measured. Before placement of the Freiburg flap into the surgical bed, 6-French afterloading catheters are threaded through the applicator and fixed into place with metal buttons. Each catheter is numbered individually (at the proximal and distal ends of the catheter) to correspond with the channels of the HDR afterloader unit. The entire flap apparatus, with catheters in place, is then positioned in the surgical bed and packed into place using lap sponges. Suturing the applicator in place is not generally necessary, assuming packing is adequate. For pelvic procedures, the bowel and ureters should be identified and protected with lead shields or retracted as far away from the applicator as safely as possible. It is important to cover the lead shields with wet gauze in order to prevent backscatter. In general, 3 lead shields ( $0.9 \mathrm{~mm}$ thick) will reduce the dose to the surrounding normal tissue by $\sim 70 \%$. In addition, when possible the distance between the source and the normal structure is increased. The prescribed dose at $1 \mathrm{~cm}, 2 \mathrm{~cm}$ and $3 \mathrm{~cm}$ is reduced to approximately $70 \%, 50 \%$, and $35 \%$, respectively. 
High dose rate-intraoperative radiation (HDR-IORT) flowchart

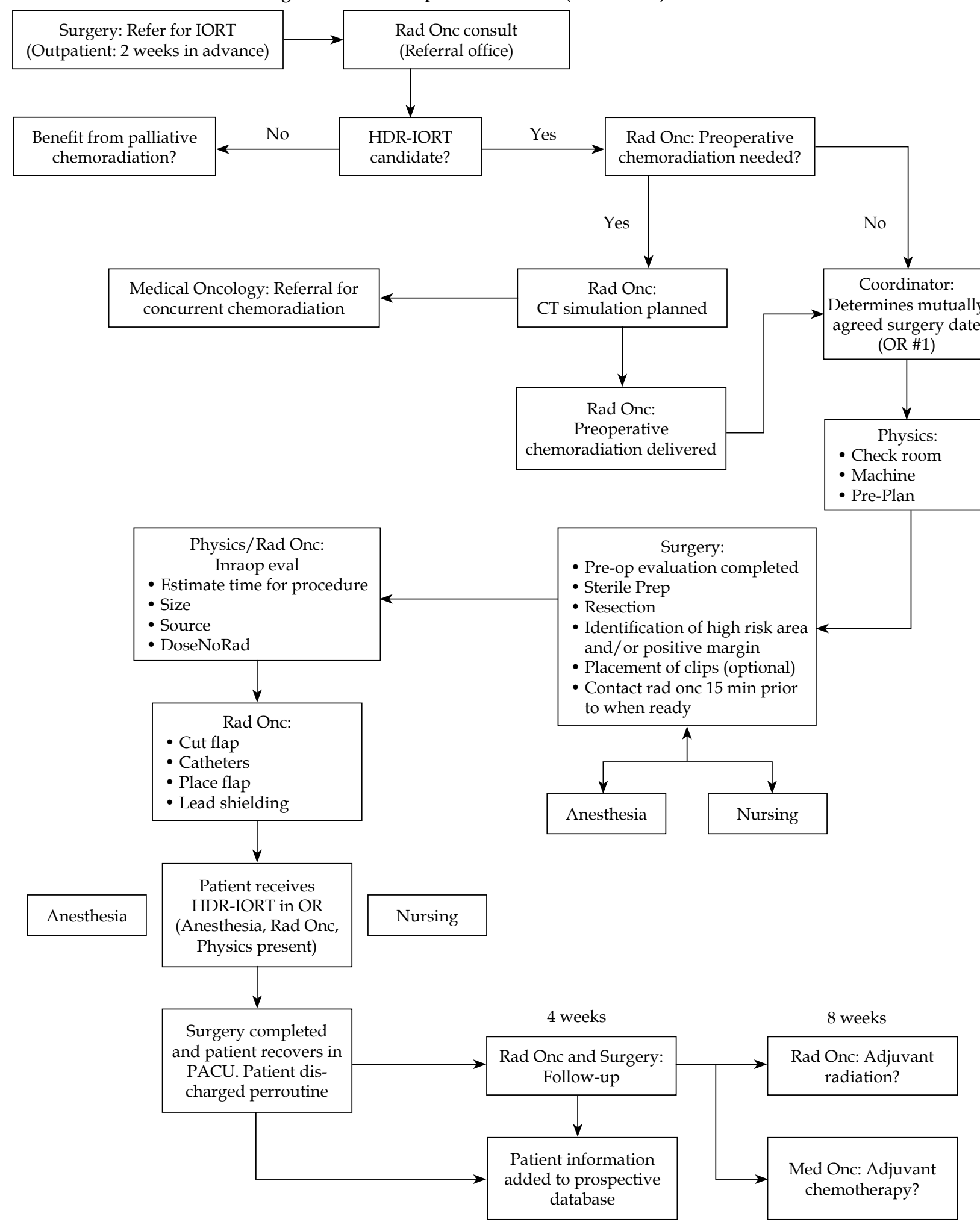

Fig. 1. An HDR-IORT flow chart shows the chronological order of treatment decisions

The dosimetric planning process at Johns Hopkins utilizes a commercially available HDR brachytherapy planning system (OncentraBrachy ${ }^{\circledR}$, Nucletron, an Elekta company, Elekta AB, Stockholm, Sweden). Prior to initiating this program, conditions affecting multiple aspects of dosimetry are determined. Due to backscatter dose enhancement from photon energies emitted by ${ }^{192} \mathrm{Ir}$, backscatter dose is equilibrated into the planning system. The flap is then con- 
Departments of Surgery, Anesthesiology, and Nursing Brachytherapy Protocol

Purposo: This protocol is a "safety chock system" to onsure that the perioperative team is ready for safo management of brachytherapy pationts in the Johns Hopkins Hospital intraoperative environment.

A. Regulatory Compliance

$\square$ Posting of safety signs and labels ("Caution Radioactive Materials")

口 Source accountability (Microselectron)

- Lead container/afterioader control box

口 Staff awareness/communication

B. Radiation Oncology Staff (radiation physicist, dosimotrist, radiation thorapist, radiation oncologist)

Consent Forms: $\square$ Surgery $\square$ ACCM $\square$ Radiation (discussion of risks/hazards)

- Notification of source implantation, including:

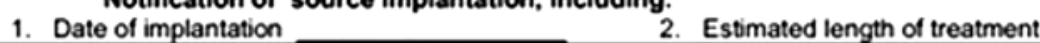

- Briefing of surgical/anesthesiology/nursing staft (patient will bo awake)

1. Approximate dose rates

2. Estimated length of treatment

3. Availability of Freiburg Flap Comments

- Radiation Exposure Survoy

들 Check expose rate measurements

D Documentation (results of area survey)

C. OR Nursing Instructions

D Check DPC/case cart for all supplies

Ensure all involved staff wear and display dosimeter badges

口. Monitor aseptic technique/maintain sterility of back table during insertion

- Notify ORAs of environmental cleanup instructions

D. OR Equipment (readily available)

$\square$ Difficult airway cart

C Crash cartdefibrillator

I Extension cords

D All necessary equipment available and functional

E. ORMIS Clinical Documentation: $\square$ Record source placement $\square$ Record OR Radiation Exposure Survoy results

F. Operating Room Attire

G. Aseptic Technique Monitored

H. Standard Procautions

I. Anesthesia Considerations

Q Patient seen in the Preoperative Evaluation Center

A Anesthesia machine pre-use check

A Alarm level outside of the OR checked

C Camera views checked ( 3 views)

C Anesthesia equipment for remote IV administration checked

J. Standard Briefing Tool

1. Identification of patient $\square$ Yes No Comments

2. Blood availability $\square$ Yes $\square$ No Comments

3. Surgical site verification $\square$ Yes $\square$ No Comments

4. Antibiotic prior to surgical incision Yes No Comments

5. Description of critical steps for the procedure and concerns $\square$

6. Discuss risks associated to the procedure

K. Dobriefing:

Problems

Comments

Attending Surgeon

OR Nurse

Attending Anesthesiologist

Radiation Oncologist

Date

Fig. 2. A safety protocol consisting of different checklists is used to ensure appropriate readiness of the perioperative team before starting HDR-IORT treatment [13]

nected to the HDR afterloader, with careful attention paid to ensure that each catheter is connected to its matching channel. Radiation is delivered by an encapsulated ${ }^{192}$ Ir source, half-life of 73 days, that is loaded via 6-French afterloading catheters (Nucletron, an Elekta company, Elekta AB, Stockholm, Sweden) into the flap once it is in place.

Every attempt should be made to minimize the dose to normal structures in the target area, including bladder, bowel, bone, and nerve roots [14]. The dose recommendations for HDR-IORT are shown in Table 2.

\section{Common challenges}

Since the development and implementation of this process, several barriers have been identified. Major areas requiring particular attention are identified below:

1) an IORT patient care coordinator who can work with each discipline can streamline the scheduling and coordinating of care,

2) all involved physicians and physicists must be present during the actual "beam on" time; residents or physician 
extenders can be used to assist with other aspects of the HDR-IORT procedure including set-up and removal,

3) good communication between the surgeon and radiation oncologist is critical for identification of the area requiring treatment,

4) be certain to obtain relevant outside medical records and previous treatment summaries prior to the procedure, especially neoadjuvant RT dose information,

5) billing compliance using appropriate reimbursement codes should be obtained with the help of the Radiation Oncology Billing and Compliance Office,

6) ensure that all required equipment is readily available and properly sterilized (ie. Flap, catheters etc.) using proper surgical and sterilization techniques,

7) comply with established safety protocols, from American Society for Radiation Oncology/American College of Radiology (ASTRO/ACR), American Brachytherapy Society (ABS), and from the institution [15].
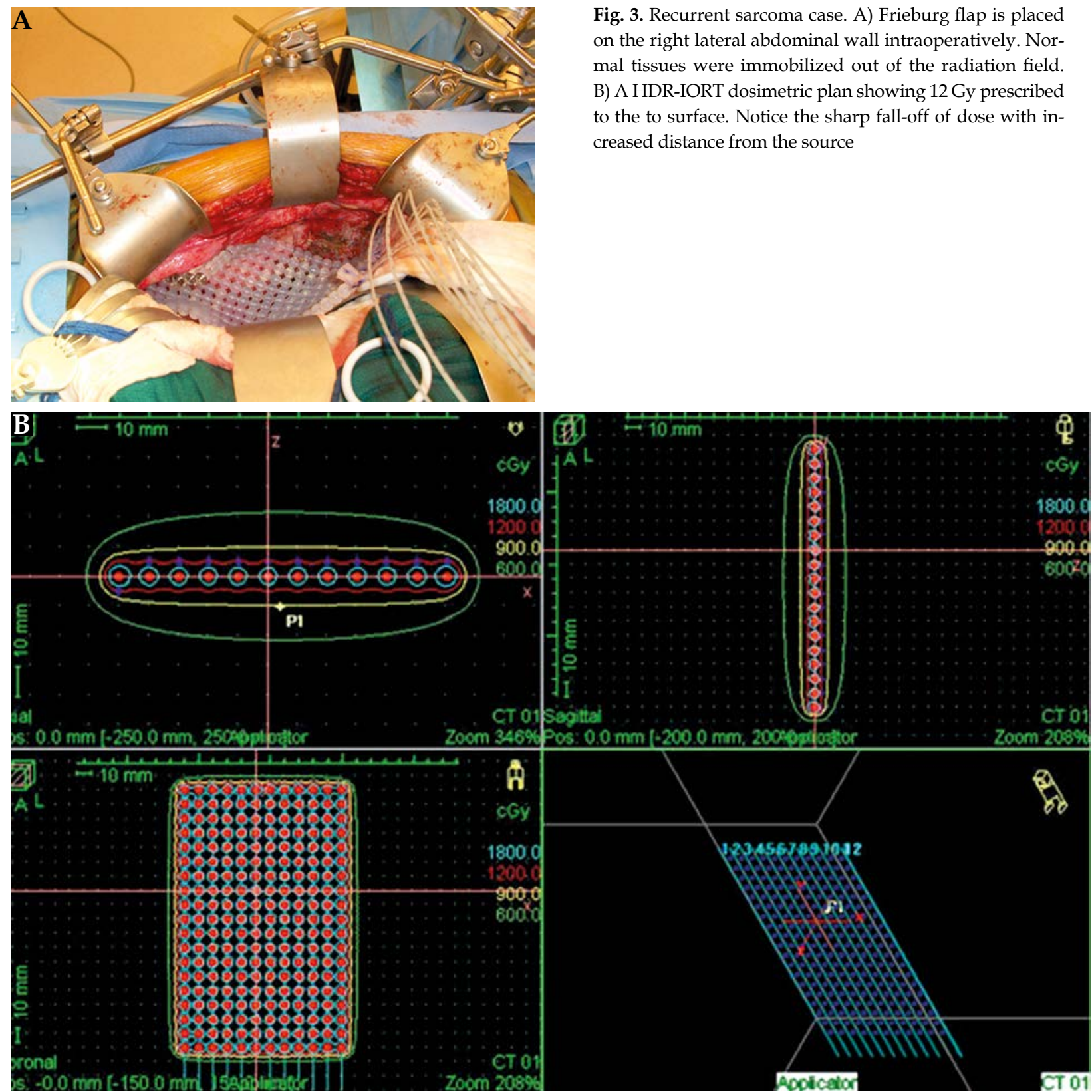

Table 2. IORT dose recommendations

\begin{tabular}{lc} 
Treatment group & $\begin{array}{c}\text { Recommended } \\
\text { IORT dose }\end{array}$ \\
\hline Following neoadjuvant therapy & $10-12 \mathrm{~Gy}$ \\
\hline $\begin{array}{l}\text { Residual microscopic disease present } \\
\text { following resection (R1 resection) }\end{array}$ & $12 \mathrm{~Gy}$ \\
\hline $\begin{array}{l}\text { Residual macroscopic disease present } \\
\text { following resection (R2 resection) }\end{array}$ & $>12 \mathrm{~Gy}$
\end{tabular}

\section{Johns Hopkins' intraoperative radiation therapy} program

Since the program's initiation in 2006, 87 patients have been treated. Of these patients, $43 \%$ had sarcomas, $26 \%$ had recurrent rectal tumors, $11 \%$ other gastrointestinal tumors, $3 \%$ genitourinary malignancies, and $17 \%$ other has steadily increased in consecutive years.

Fig. 3. Recurrent sarcoma case. A) Frieburg flap is placed on the right lateral abdominal wall intraoperatively. Normal tissues were immobilized out of the radiation field. B) A HDR-IORT dosimetric plan showing 12 Gy prescribed to the to surface. Notice the sharp fall-off of dose with increased distance from the source tumor types. The number of patients undergoing IORT 


\section{Cases}

The following cases highlight the utilization of HDRIORT and its potential to decrease the risk of a local recurrence.

\section{Case 1. Recurrent sarcoma}

A 35-year-old nonsmoking male with no significant past medical history and no family history of colon cancer presented with increasing right-sided abdominal pain and a protuberant mass on the right side of his abdomen. A computed tomography (CT) scan confirmed a large mass located inferior to the right kidney and adjacent to the liver. Pathology showed a well-differentiated retroperitoneal liposarcoma with negative margins and lymph nodes.

Seven months following resection, a CT scan showed possible extra-peritoneal recurrence in the right lobe of the liver, and was biopsy-confirmed liposarcoma. A partial abdominal wall resection of the tumor was performed and HDR-IORT was delivered to a dose of $12 \mathrm{~Gy}$ to a $36 \mathrm{~cm}^{2}$ area of the right abdominal wall (Figs. 3A,B). All margins were negative and pathology again demonstrated a well-differentiated liposarcoma. Clips were plac-
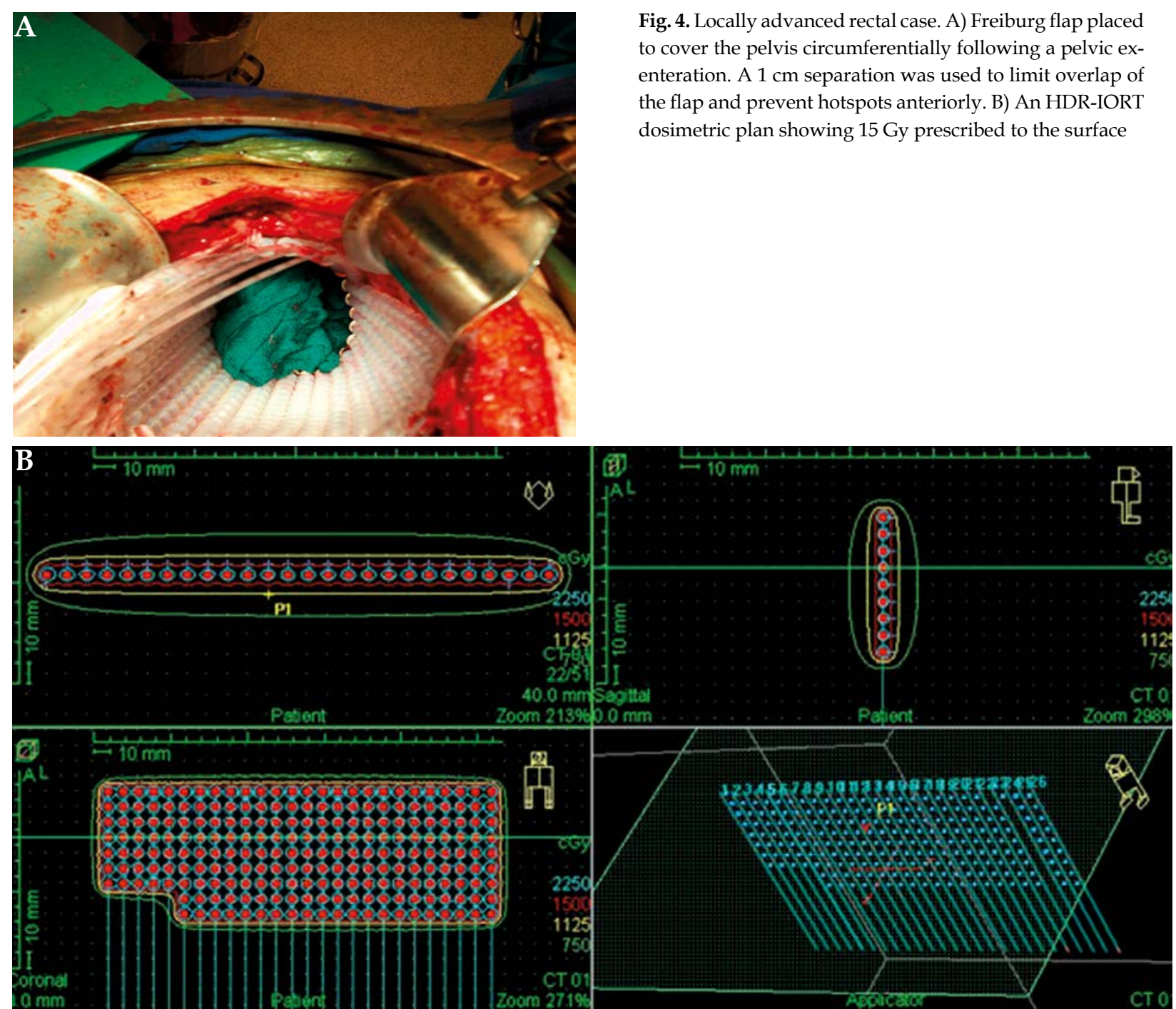

ed to outline the tumor bed. EBRT was given to the patient following surgery, to a total dose of $45 \mathrm{~Gy}$ in $1.8 \mathrm{~Gy}$ fractions delivered to the right chest and abdominal wall. Side effects from EBRT included moderate skin irritation, mild fatigue, and some discomfort in the right upper quadrant. Almost 3 years after surgery and RT, a CT scan revealed some increased soft tissue stranding in the retroperitoneum and biopsy confirmed a recurrent retroperitoneal liposarcoma in the inferior right retroperitoneal area outside of the previous treatment field area. The recurrent mass was excised along with the right kidney and portions of the small bowel; an HDR-IORT dose of 12 Gy was delivered to this area followed by EBRT of 39.6 Gy in 22 fractions. The patient has been doing well since then with no new recurrences or complications with a follow-up time of 66 months.

\section{Case 2. Locally advanced rectal cancer}

A 39-year-old male with a family history significant for colon cancer and no significant past medical history presented with unintentional weight loss and a complete gastrointestinal obstruction. Based on imaging, the patient was diagnosed with locally advanced rectal cancer,

Fig. 4. Locally advanced rectal case. A) Freiburg flap placed to cover the pelvis circumferentially following a pelvic exenteration. A $1 \mathrm{~cm}$ separation was used to limit overlap of the flap and prevent hotspots anteriorly. B) An HDR-IORT dosimetric plan showing 15 Gy prescribed to the surface 
$2.5 \mathrm{~cm}$ from the anal verge located in the anterior aspect of the rectal vault.

The patient received chemotherapy, consisting of 5-FU, and EBRT to a dose of $54 \mathrm{~Gy}$ to the pelvis. Subsequent CT scans demonstrated disease progression and involvement of nearby structures, including the prostate, seminal vesicles, and anal sphincter. Also noted was circumferential extension to the pelvic sidewall. The patient then underwent additional chemotherapy, consisting of FOLFIRINOX, complicated by the development of acute renal failure. Due to the local progression of the tumor, a total pelvic exenteration was performed along with administration of HDR-IORT. An HDR-IORT dose of 15 Gy was delivered to the surface of the entire pelvis (Figs. 4A,B). The patient did well post-operatively and has had no further complications.

\section{Conclusions}

This article provides an overview of the requirements for the initiation and operation of a successful HDR-IORT program. HDR-IORT was the focus of this article as it has some potential benefits when compared to IOERT. For example, HDR-IORT has the ability to deliver focused RT in areas where an IOERT cone is not suitable. However, having both HDR-IORT and IOERT provides optimal flexibility for a wide range of treatment sites. When planning the initiation of an HDR-IORT program, it can be helpful to seek advice from an experienced, pre-existing program and, when possible, receive hands-on training. Every case is unique and requires multidisciplinary management to ensure safe and accurate treatment. Furthermore, institutions with established IORT programs may benefit from collaborating with institutions developing new IORT programs and provide support and guidance via educational workshops.

With improved systemic therapy, local control will continue to gain importance and IORT will likely play a larger role in the management of locally advanced primary and recurrent tumors [5-7,9]. Furthermore, ongoing advances in translational research can lead to increased use of radiation sensitizers and radio-protectors in conjunction with IORT [16]. While retrospective analyses have documented improvements in multiple outcomes using IORT, prospective multicenter studies are still needed to determine its true efficacy $[5-7,10]$.

\section{Research support for the study}

Claudio X. Gonzalez Family Foundation.

\section{Disclosure}

Authors report no conflict of interest.

\section{References}

1. Willett CG, Czito BG, Tyler DS. Intraoperative Radiation Therapy. J Clin Oncol 2007; 25: 971-977.

2. Alfieri S, Morganti AG, Giorgio AD et al. Improved survival and local control after intraoperative radiation therapy and postoperative radiotherapy: a multivariate analysis of $46 \mathrm{pa}-$ tients undergoing surgery for pancreatic head cancer. Arch Surg 2001; 136: 343-347.
3. Schuricht AL, Spitz F, Barbot D et al. Intraoperative radiotherapy in the combined-modality management of pancreatic cancer. Am Surg 1998; 64: 1043-1049.

4. Niewald $\mathrm{M}$, Fleckenstein J, Licht $\mathrm{N}$ et al. Intraoperative radiotherapy combined with external beam radiotherapy for soft-tissue sarcomas - a retrospective evaluation of the Homburg experience in the years 1995-2007. Radiat Oncol 2009; 4: 32-38.

5. Roth TM, Secord AA, Havrilesky LJ et al. High dose rate intraoperative radiotherapy for recurrent cervical cancer and nodal disease. Gynecol Oncol 2003; 91: 258-260.

6. Harrison LB, Minsky BD, Enker WE et al. High dose rate intraoperative radiation therapy (HDR-IORT) as a part of management strategy for local advanced primary and recurrent rectal cancer. Int. J Radiat Oncol Biol Phys 1998; 42: 325-330.

7. Pawlik TM, Ahuja N, Herman JM. Radiation in retroperitoneal sarcomas. Curr Opin Oncol 2007; 4: 359-366.

8. Calvo FA, Meirino RM, Orecchia R. Intraoperative radiation therapy. First part: Rationale and techniques. Critical Reviews in Oncology/Hematology 2006; 59: 106-115.

9. Calvo FA, Meirino RM, Orecchia R. Intraoperative radiotherapy. Part 2. Clinical results. Critical Reviews in Oncology/Hematology 2006; 59: 116-127.

10. Gunderson LL. Clinical Radiation Oncology. Saunders/Elsevier, Philadelphia 2011; pp. 315-327.

11. Calvo FA, Sole CV, Herranz R et al. Intraoperative radiotherapy with electrons: fundamentals, results and innovation. Ecancermedialscience 2013; 7: 339.

12. Gao S, Delclos ME, Tomas LC et al. High-dose-rate remote afterloaders for intraoperative radiation therapy. AORN 2007; 86: 827-836.

13. Rodriguez-Paz JM, Mark LJ, Herzer KR et al. A novel process for introducing a new intraoperative program: a multidisciplinary paradigm for mitigating hazards and improving patient safety. Anesthesia and Analgesia 2009; 108: 202-210.

14. Nocolaou N. Prevention and management of radiation toxicity. Skin 2004; 5: 6-500.

15. Nag S, Shasha D, Janjan N et al. The American Brachytherapy Society recommendations for brachytherapy of soft tissue sarcomas. Int J Radiat Oncol Biol Phys 2001; 49: 1033-1043.

16. Gunderson LL, Willett CG, Calvo FA et al. Intraoperative Radiation: Techniques and Results. Humana Press, New York 2011; pp. 37-39. 